\section{Method of silver impregnation for nervous tissue embedded in paraffin}

\section{SERGE DUCKETT ${ }^{1}$ From Maida Vale Hospital for Nervous Diseases, London}

The aim of the silver impregnation methods for paraffinembedded sections of nervous tissue is to combine finesse of histological detail with an easy method of processing.

The following method is presented here because of its rapidity, reliability, staining quality, and utility as a general purpose stain. The basic technique, without the variations, was devised by Fincher (1932) on the basis of Hortega's silver carbonate impregnation method for astrocytes and its purpose was to stain cerebral tumours.

\section{METHOD}

1 Paraffin sections, of 5 to $8 \mu$ for pathological studies and of 10 to $20 \mu$ for anatomical studies, are mounted on albuminized slides a few hours before use. The sections are then slowly brought through xylol (5 minutes), absolute alcohol (5 minutes), and alcohol $95^{\circ}$ (5 minutes), and washed in distilled water.

2 Mordant in the following solution at $60^{\circ} \mathrm{C}$. until yellow.

Silver nitrate $2 \% \ldots \ldots \ldots \ldots \ldots . .30 \mathrm{ml}$.

Pyridine $\quad \ldots \ldots \ldots \ldots \ldots \ldots \ldots$............ drops

Absolute alcohol $\ldots \ldots \ldots \ldots \ldots \ldots 15$ drops

This usually takes 30 minutes. I prefer not to pre-warm my solution. Prolonged mordanting gives too dark a picture.

3 Wash quickly in $95^{\circ}$ alcohol.

Received for publication 31 March 1964.

'Presently clinical fellow with the Medical Research Council.

\section{A simple constant-infusion apparatus-continued.}

At present two of these models are being used for the injection of tritiated thymidine into rats at a rate of $0.5 \mathrm{ml}$. per 25 hours (Foot, 1963).

The advantages of the present device are its simplicity cheapness, accuracy, 'foolproofness', and the minute amounts that can be injected. Its working can also be checked easily by observing the rotation of the micrometer head. Its disadvantage is that a separate motor is required for each different speed but these are cheap and can be changed in a few moments.

The author is in receipt of a grant from the British Empire Cancer Campaign.

\section{REFERENCES}

Foot, E. C. (1963). Nature (Lond.), 198, 297.
4 Impregnate in the following solution at $60^{\circ} \mathrm{C}$. until a dark amber colour. If the solution has been pre-warmed this takes from 15 to 30 minutes; if not it may take up to an hour, at which time the section should be taken out no matter what colour it is.

Hortega's strong silver carbonate (lithium or sodium) $)^{2} \ldots \ldots \ldots \ldots . \ldots \mathrm{ml}$.

Pyridine $\quad \ldots \ldots \ldots \ldots \ldots \ldots \ldots$........... drops

Absolute alcohol ...............15 drops

5 Wash rapidly in $95^{\circ}$ alcohol.

6 Reduce in $10 \%$ formol for one to two minutes.

7 Wash in distilled water.

8 If desired tone in a $\mathbf{0 . 2} \%$ gold chloride solution.

9 Wash in distilled water.

10 Leave in a $2 \%$ oxalic acid solution for a few minutes until the sections become reddish. This step accentuates the staining of axones.

11 Fix, if desired, in a $5 \%$ solution of sodium thiosulphate.

12 Wash in distilled water, dehydrate, clear, and mount in balsam.

2a Lithium carbonate solution To $5 \mathrm{ml} .10 \%$ silver nitrate add $20 \mathrm{ml}$. of a saturated solution of lithium carbonate and then drop by drop add $28 \%$ ammonia water barely to dissolve the precipitate. Bring volume to $75 \mathrm{ml}$. with distilled water and filter. Keep in brown bottle. b Sodium carbonate solution To $5 \mathrm{ml} .10 \%$ silver nitrate add $20 \mathrm{ml}$. $5 \%$ sodium carbonate solution and then drop by drop add $28 \%$ ammonia water barely to dissolve the precipitate. Add about $20 \mathrm{ml}$. distilled water and filter, bringing volume to $45 \mathrm{ml}$. Keep in brown bottle.

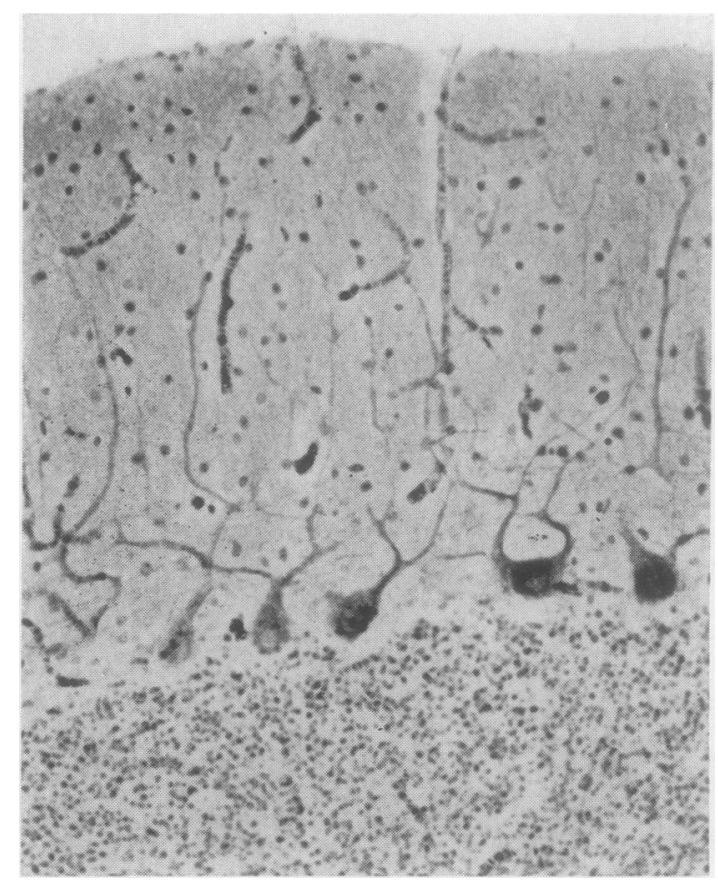

FIG. 1. Normal Purkinje cells, and horizontal fibres of basket cells. Variant $4 \times 120$ (mordanting, one hour, and impregnation one hour). 
TABLE I

PROCEDURE FOR VARIANTS OF THE METHOD

Step Variant

\begin{tabular}{lllllll}
\hline 1 & 2 & 3 & 4 & 5 & 6
\end{tabular}

Mount on albuminized slides, leave to dry for 12 hours and deparaffinize

Mordant in $2 \%$ silver nitrate solution until yellow, usually about 30 minutes, then wash in $95^{\circ}$ alcohol.

Impregnate with Hortega's silver carbonate (lithium) solution. Impregnate with Hortega's silver carbonate (sodium) solution

Reduce in $10 \%$ formol, then wash in water

Fix in $5 \%$ hypo Tone in $0.2 \% \mathrm{AuCl}$

Impregnate with Hortega's silver carbonate
Fix in $5 \%$ hypo formol, then wash in water

Clear and mount $\quad$ Fix in $5 \%$ hypo $\quad$ Wash in water then

leave in $2 \%$ oxalic

Tone in $0.2 \% \mathrm{AuCl}$

Clear and mount

Fix in $5 \%$ hypo

Wash in water then

leave in $2 \%$ oxalic acid

7

8

Dehydrate, clear, and Fix in $5 \%$ hypo

Dehydrate, clear, and mount

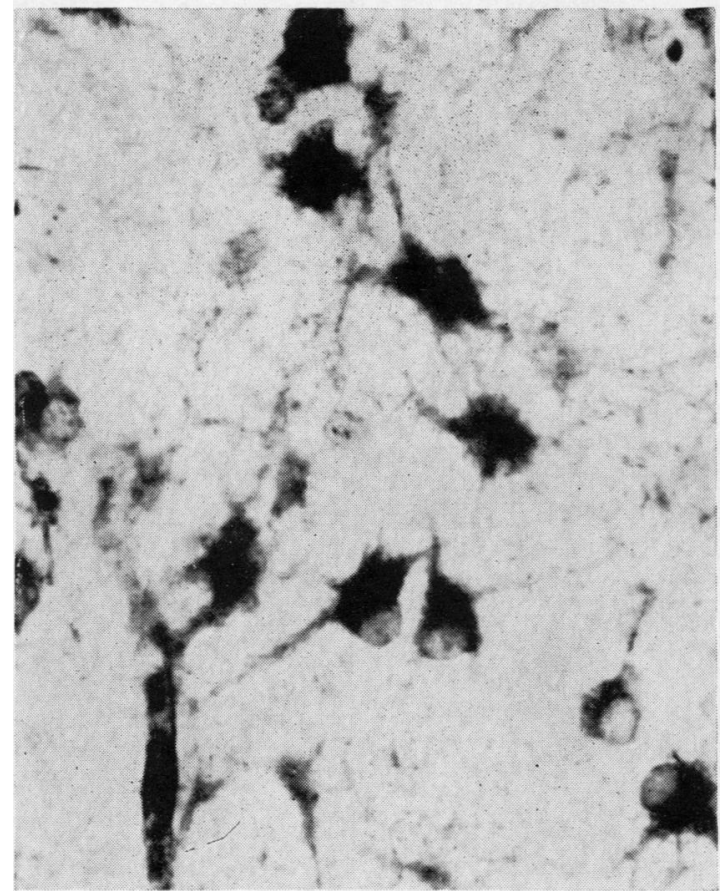

FIG. 2

FIG. 2. Hypertrophied oligodendrocytes in cerebral white matter. Variant $2 \times 400$ (mordanting 45 minutes; impregnation 45 minutes).

FIG. 3. Climbing fibres ascending through the granular layer and surrounding the base of the Purkinje cells. Above are seen the horizontal fibres of the basket cells. Variant 2 $\times \mathbf{4 0 0}$ (mordanting half an hour; impregnation half an hour).

\section{VARIANTS ON THE METHOD}

There are a number of variants to this technique which when employed bring out different morphological features of the section (Table I). These are divided into two groups, the first one uses Hortega's strong lithium silver carbonate solution, the second Hortega's strong 


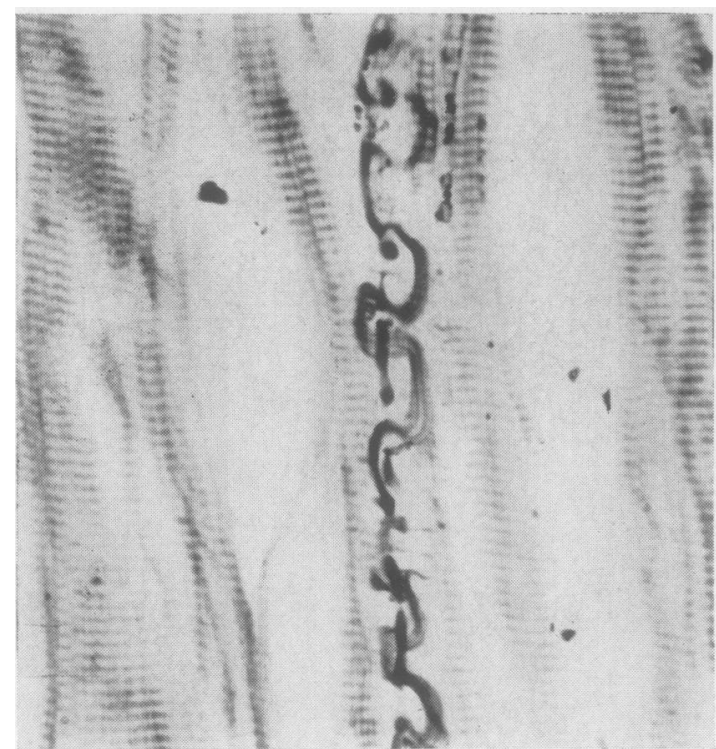

FIG. 4. Nerve fibre coursing through muscle fibres. Variant $3 \times 400$ (mordanting half an hour; impregnation half an hour).

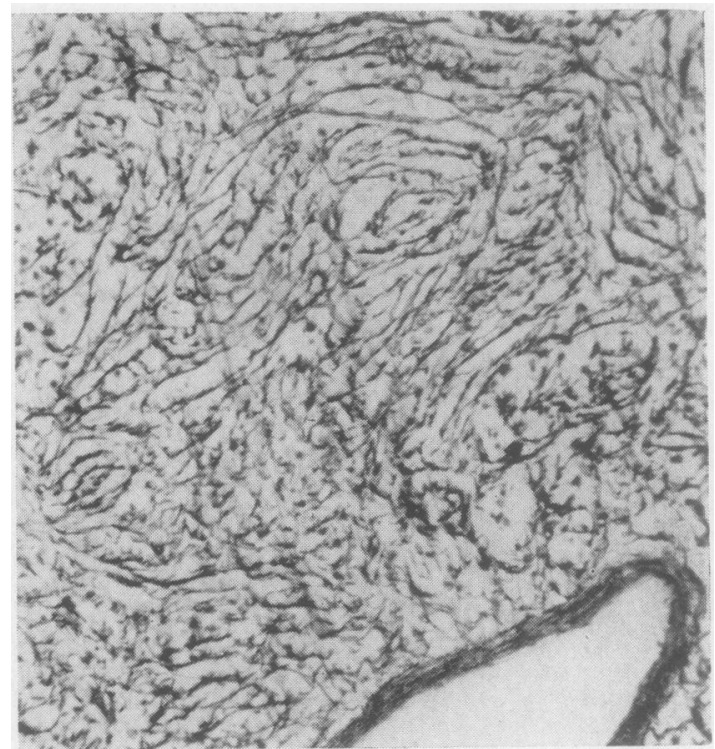

FIG. 5. The meshwork of reticulin in a meningioma. Variant $5 \times 120$ (mordanting for one hour; impregnation 40 minutes).

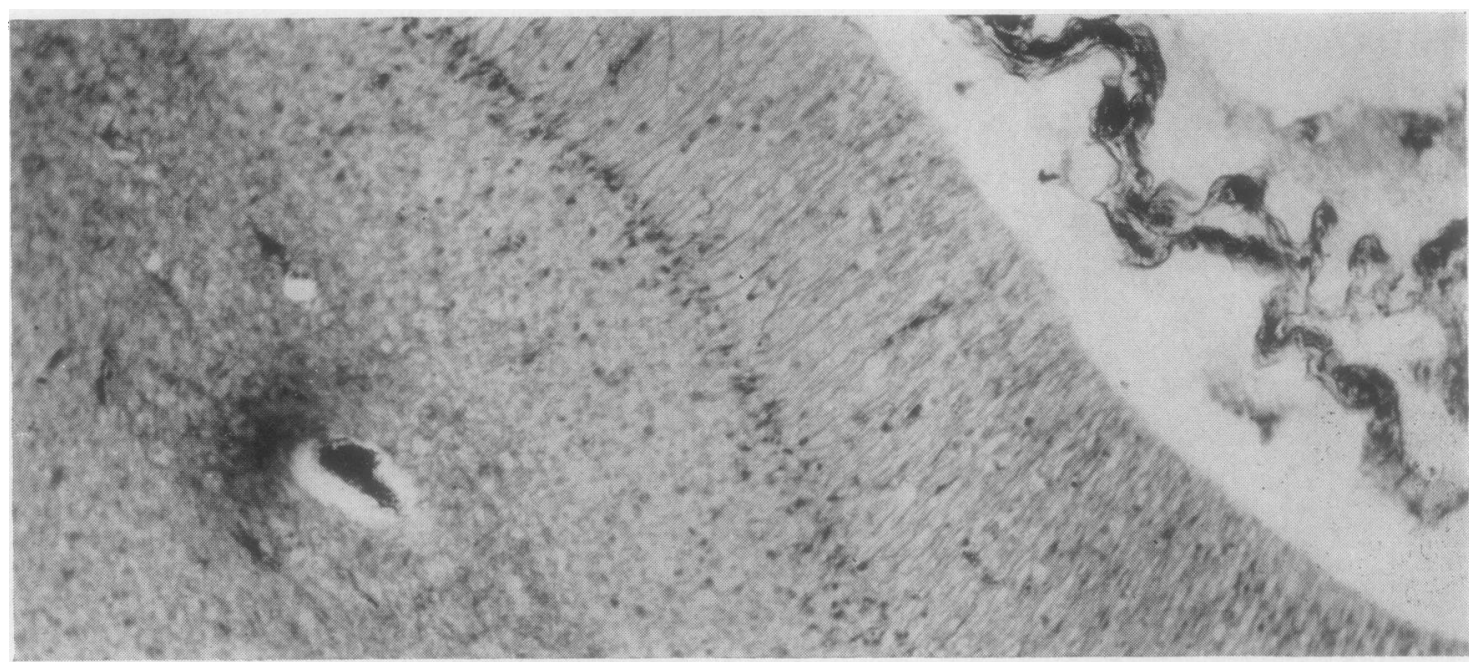

FIG. 6. Isomorphic gliosis in the cerebellum. Variant $6 \times 120$ (mordanting for half an hour; impregnation for half an hour).

reduced in formol and toned with gold chloride. Variants 3 and 6 are reduced in formol, toned in gold chloride, washed in $2 \%$ oxalic acid, dehydrated and mounted in balsam.

Variants 1 and 4, the untoned sections, show well the axons and glial fibres (Fig. 1). Variant 2 shows the glia (Fig. 2), the more delicate fibrillary systems (Fig. 3), and neurons. Variant 3 shows the axons well, and has been useful in showing the nerve fibres coursing through muscle (Fig. 4). Variant 5 is a reticulin stain (Fig. 5).
Variant 6 also shows the reticulin, as reddish fibres against a grey background, and gliosis (Fig. 6). With all these variants the staining may benefit from varying the times for mordanting and impregnating, and the reducing formol solution can be diluted to $1 \%, 5 \%$, or $20 \%$.

I would like to thank Drs. W. McMenemey, A. G. E. Pearse, C. Margolis, and John Prineas for their help.

\section{REFERENCES}

Fincher, E. F. Jr. (1932). Amer. J. Cancer., 16, p. 918. 